\title{
Lumbar paravertebral blockade as intractable pain management method in palliative care
}

This article was published in the following Dove Press journal:

OncoTargets and Therapy

2 September 2013

Number of times this article has been viewed

\author{
Iwona Zaporowska- \\ Stachowiak ${ }^{1,2}$ \\ Aleksandra Kotlinska- \\ Lemieszek $^{3}$ \\ Grzegorz Kowalski ${ }^{3}$ \\ Katarzyna Kosicka ${ }^{4}$ \\ Karolina Hoffmann ${ }^{5}$ \\ Franciszek Główka ${ }^{4}$ \\ Jacek Łuczak ${ }^{2}$ \\ 'Department of Pharmacology, \\ ${ }^{2}$ Palliative Medicine In-patient \\ Unit, University Hospital of Lord's \\ Transfiguration, ${ }^{3}$ Department \\ of Palliative Care, ${ }^{4}$ Department \\ of Physical Pharmacy and \\ Pharmacokinetics, ${ }^{5}$ Department \\ of Internal Medicine, Metabolic \\ Disorders and Arterial Hypertension, \\ Poznań University of Medical Sciences, \\ Poznań, Poland
}

Correspondence: Iwona ZaporowskaStachowiak

Poznań University of Medical Sciences,

Chair and Department of Pharmacology,

Rokietnicka 25A, 60-806 Poznań, Poland

Tel +48609267073

Email iwozapor@amp.edu.pl
Abstract: Optimal symptoms control in advanced cancer disease, with refractory to conventional pain treatment, needs an interventional procedure. This paper presents coadministration of local anesthetic (LA) via paravertebral blockade (PVB) as the alternative to an unsuccessful subcutaneous fentanyl pain control in a 71-year old cancer patient with pathological fracture of femoral neck, bone metastases, and contraindications to morphine. Bupivacaine in continuous infusion $(0.25 \%$, $5 \mathrm{~mL} \cdot$ hour $^{-1}$ ) or in boluses ( $10 \mathrm{~mL}$ of $0.125 \%-0.5 \%$ solution $)$, used for lumbar PVB, resulted in pain relief, decreased demand for opioids, and led to better social interactions. The factors contributing to an increased risk of systemic toxicity from LA in the patient were: renal impairment; heart failure; hypoalbuminemia; hypocalcemia; and a complex therapy with possible drug-drug interactions. These factors were taken into consideration during treatment. Bupivacaine's side effects were absent. Coadministered drugs could mask LA's toxicity. Elevated plasma $\alpha 1$-acid glycoprotein levels were a protective factor. To evaluate the benefit-risk ratio of the PVB treatment in boluses and in constant infusion, bupivacaine serum levels were determined and the drug plasma half-lives were calculated. Bupivacaine's elimination was slower when administered in constant infusion than in boluses ( $\mathrm{t}_{1 / 2}=7.80$ hours versus 2.64 hours). Total drug serum concentrations remained within the safe ranges during the whole treatment course $\left(22.9-927.4 \mathrm{ng} \cdot \mathrm{mL}^{-1}\right)$. In the case presented, lumbar PVB with bupivacaine in boluses $\left(\leq 137.5 \mathrm{mg} \cdot 24\right.$ hours $\left.^{-1}\right)$ was an easy to perform, safe, effective method for pain control. Bupivacaine in continuous infusion ( $\left.\leq 150 \mathrm{mg} \cdot 12 \mathrm{hours}^{-1}\right)$ had an acceptable risk-benefits ratio, but was ineffective.

Keywords: bone metastases, bupivacaine, intractable pain, opioid ineffectiveness

\section{Introduction}

The expectations towards quality of life in palliative patients have increased recently. A satisfactory pain control positively affects physical, emotional, and spiritual aspects of patients' lives.

As opioid dose augmentation may be restricted by the drug's adverse effects, the alternative way of pain relief is peripheral nerve blockade. According to Richardson et al, Hugo Salheim performed the first paravertebral blockade (PVB) in 1905. ${ }^{1}$ Ipsilateral, prolonged, somatic, and sympathetic nerve blockade is usually spread above and below over a few dermatomes after a single, percutaneous, large-volume (5-30 mL) local anesthetic (LA) injection into the paravertebral space. ${ }^{2,3}$ Long-acting bupivacaine $(0.125 \%-0.5 \%)$, ropivacaine $(0.2 \%-1 \%)$, mepivacaine $(2 \%)$, or faster eliminated, less cardiotoxic lidocaine (1\%) are used for PVBs.

After about 50 years of falling out of favor, percutaneous catheterization was renewed and the specific method was detailed for adults: by Moore (obstetrics); ${ }^{4}$ Eason and Wyatt 
(pain control after multiple rib fractures or thoracotomy) ${ }^{5}$ or modified by Lonnqvist for children. ${ }^{6-8}$ Uni- and bilateral PVB has been used for both acute ${ }^{9-16}$ and chronic pain in adults ${ }^{17-21}$ and in children for intra- and postoperative analgesia, ${ }^{6,22}$ but is still relatively rarely used in the palliative care. ${ }^{23,24}$ The percentage of PVBs' failure $(6.1 \%-10 \%$ in prospective trials on adults and children) may reflect obstacles in spotting the paravertebral space compared to epidural or subarachnoid spaces. ${ }^{25,26}$ PVB brings lower risks of hypotension, headache, nausea, vomiting, Horner's syndrome, pulmonary complications, urinary retention, aspiration of cerebrospinal fluid, and volume and pressure effect than the spinal or epidural anesthesia (EDA), ${ }^{2,25,27,28}$ providing for similar pain scores. ${ }^{15,29,30}$ PVB is superior to EDA in patients with coagulopathy or under anticoagulant treatment, with anatomical abnormalities (scoliosis, spina bifida), infection (systemic or at the site of injection), hypovolemia (not severe), as well as central nervous system (CNS) or cardiovascular diseases (affecting the cardiac output), which contraindicates the use of EDA. ${ }^{31}$ The aim of PVB is a LA action at the site of administration, but the drug may spread medially to the epidural, or laterally to the intercostal space, below or above to the contiguous levels in high interpatient variability. ${ }^{21,32-34} \mathrm{~A}$ larger volume of a LA solution produces anesthesia over a larger number of dermatomes. ${ }^{1}$ As a result of an inadvertent direct vascular injection or rapid absorption of the LA solution, the drug may also reach the systemic circulation and, potentially, induce central nervous and/or cardiovascular system toxicity. Older patients, with hypoproteinemia, liver impairment, heart- or renal failure, are at higher risk of LA toxicity. We report the case of a successful lumbar PVB with bupivacaine in a 71-year-old palliative patient with breast cancer suffering from intractable right leg pain generated by pathological fracture of femoral neck, bone metastases, and degenerative changes in the spinal cord and symptoms of opioid toxicity. Renal impairment, heart failure, hypoalbuminemia, contraindications to EDA, and a complex therapy creating the risk of drug-drug interactions, required consideration.

\section{Case report}

A 71-year-old woman was referred to the palliative care unit of the Poznań Palium Hospice because of the poor pain control of her L-S (lumbar to sacral) backbone radiating to both legs. Just before the admission to the hospice, her severe pain (of bone and neuropathic origin) was poorly controlled, despite having been treated with fentanyl TD $75 \mu \mathrm{g} \cdot$ hour $^{-1}$, plus morphine sulfate (MF) subcutaneous (SC) (doses every 4 hours) $40 \mathrm{mg}$ daily, and paracetamol 1,500 mg orally (po) On admission, the pain was assessed as 7-8/10 in rest, while the breakthrough pain equaled $10 / 10$ according to verbal rating scale (VRS) (see Methods section). The physical findings during this hospice examination revealed skin and mucous membrane pallor, mycosis of the mucous membrane in the mouth, decreased muscle tone, and partial paresis of both legs, particularly escalated on the right side. Table 1 presents clinical characteristics of the patient.

\section{Pertinent medical history}

The primary site of cancer disease was the right breast. The patient had a mastectomy, followed by chemotherapy. Metastases to the lung led to a right, upper lobectomy with lymphadenotomy, and chemotherapy. Metastases to the bones (vertebral column: Th11-12, L5) and lymph nodes (of aortic arch and paratracheal lymph nodes) were detected and palliative radiotherapy to Th11-12, L5 region was performed. Extraspinal metastases were also detected (to the right and left hip bone shafts, right ilium, and hip joint cavity). Pathological fracture of right femoral neck had impaired pain control. For many years, the patient has been diagnosed with multiple degenerative changes in the Th12L5 segment, essential hypertension (well controlled with pharmacotherapy), New York Heart Association (NYHA) class II heart failure (relative circulatory efficiency state), chronic kidney disease (CKD; stage 3, estimated glomerular filtration rate $[\mathrm{eGFR}]=43 \mathrm{~mL} / \mathrm{min} / 1.73 \mathrm{~m}^{2}$ ), and depression (no written data/documentation available; history taking difficult due to patient's confused state). The patient declared no drug allergies.

\section{Patient's treatment in hospice}

The results of laboratory investigations on the patient's admission and during hospitalization are presented in Table 2. As a result of impaired renal function, the regular therapy with MF was switched to fentanyl 750-1,250 $\mu$ g daily in constant

Table I Clinical characteristics of the presented patient

\begin{tabular}{lll}
\hline Factor & On admission & During hospitalization \\
\hline Age (years) & $7 \mathrm{I}$ & \\
BMI & 23.40 & 22.34 \\
Karnofsky score & 40 & $50-10$ \\
ECOG score & 4 & $4-2$ \\
MiniMental score & 4 & 18 \\
BPs (mmHg) & 88 & $116.3-133.64$ \\
BPd $(\mathrm{mmHg})$ & 59 & $70.50-74.64$ \\
Heart rate $(\mathrm{I} / \mathrm{min})$ & 76 & $86.18-95.25$ \\
eGFR $\left(\mathrm{mL} / \mathrm{min} / \mathrm{I} .73 \mathrm{~m}^{2}\right)^{*}$ & 21.01 & 31.72 \\
\hline
\end{tabular}

Note: *eGFR was calculated by short MDRD.

Abbreviations: BMI, body mass index; BPd, diastolic blood pressure; BPs, systolic blood pressure; ECOG score, Eastern Cooperative Oncology Group scale performance score; eGFR, epidermal growth factor receptor; MDRD, Modification of Diet in Renal Disease. 
Table 2 The results of laboratory investigations on patient's admission and during hospitalization

\begin{tabular}{lll}
\hline $\begin{array}{l}\text { Laboratory } \\
\text { investigations }\end{array}$ & On admission & $\begin{array}{l}\text { During } \\
\text { hospitalization }\end{array}$ \\
\hline Albuminemia $(g / L)$ & 22.80 & $20.70-16.40$ \\
Creatinine in serum $(\mu \mathrm{M})$ & 283.50 & $255.50-198.20$ \\
Urea in serum $(\mathrm{mM})$ & 27.70 & $29.45-14.68$ \\
Ca in serum $(\mathrm{mM})$ & 1.90 & $1.90-2.03$ \\
Hb $(\mathrm{mM})$ & 6.20 & $5.20-5.80$ \\
HTC & 0.320 & $0.295-0.256$ \\
PT time (seconds) & & 14.00 \\
PT indicator $(\%)$ & & 75.70 \\
INR & & 1.32 \\
$\alpha I$-acid glycoprotein & & $1.78-2.65$ \\
in plasma $(g / L)$ & & \\
Urine & & Proteinuria $(0.3 \mathrm{~g} / \mathrm{L})$, \\
& & leukocyturia \\
\hline
\end{tabular}

Abbreviations: INR, international normalized ratio; Hb, hemoglobin; HTC, hematocrit; PT, prothrombin.

SC infusion. Within 2 days, this improved the patient's mental state incredibly but had a minor effect on the pain relief, despite repeated MF rescue doses (Figure 1). Pamidronate was not introduced because of low calcium and high creatinine serum concentrations. Because of poor pain control, EDA was considered. However, the international normalized ratio (INR) value indicated an increased risk of bleeding or hematoma and was a contraindication to the EDA. Next, PVB with bupivacaine solution in boluses was performed with an excellent result. To lower the incidence of nursing procedures (waking up/disturbing/bothering the patient), the drug's administration was switched into constant paravertebral infusion. The effect on pain was poorer and bupivacaine serum concentrations increased, in comparison to boluses. Thus, after one day, we decided to withdraw bupivacaine for the following 16 hours. Then, LA boluses were reintroduced with an excellent effect (Figures 2 and 3).

\section{Methods}

The study was approved by the Bioethical Committee at the Poznań University of Medical Sciences and written, informed consent was obtained from the patient. Lumbar percutaneous PVB was performed.

Pain was rated by the patient with the use of a $0-10$ verbal rating scale (VRS; $0=$ no pain, $10=$ worst imaginable pain).

Premedication before PVB: Midazolam, $2 \mathrm{mg}$ intravenous (IV).

\section{Technique of performing a percutaneous paravertebral lumbar block (LPVB)}

Under aseptic condition, the patient, in lateral position, lay on one side, with the blocked side upwards. The skin area was prepared with a Betadine ${ }^{\circledR}$ (Purdue Pharma L.P., Stamford, CT, USA) solution. The level of the spinous process (L2) was determined. The point of the needle insertion was set

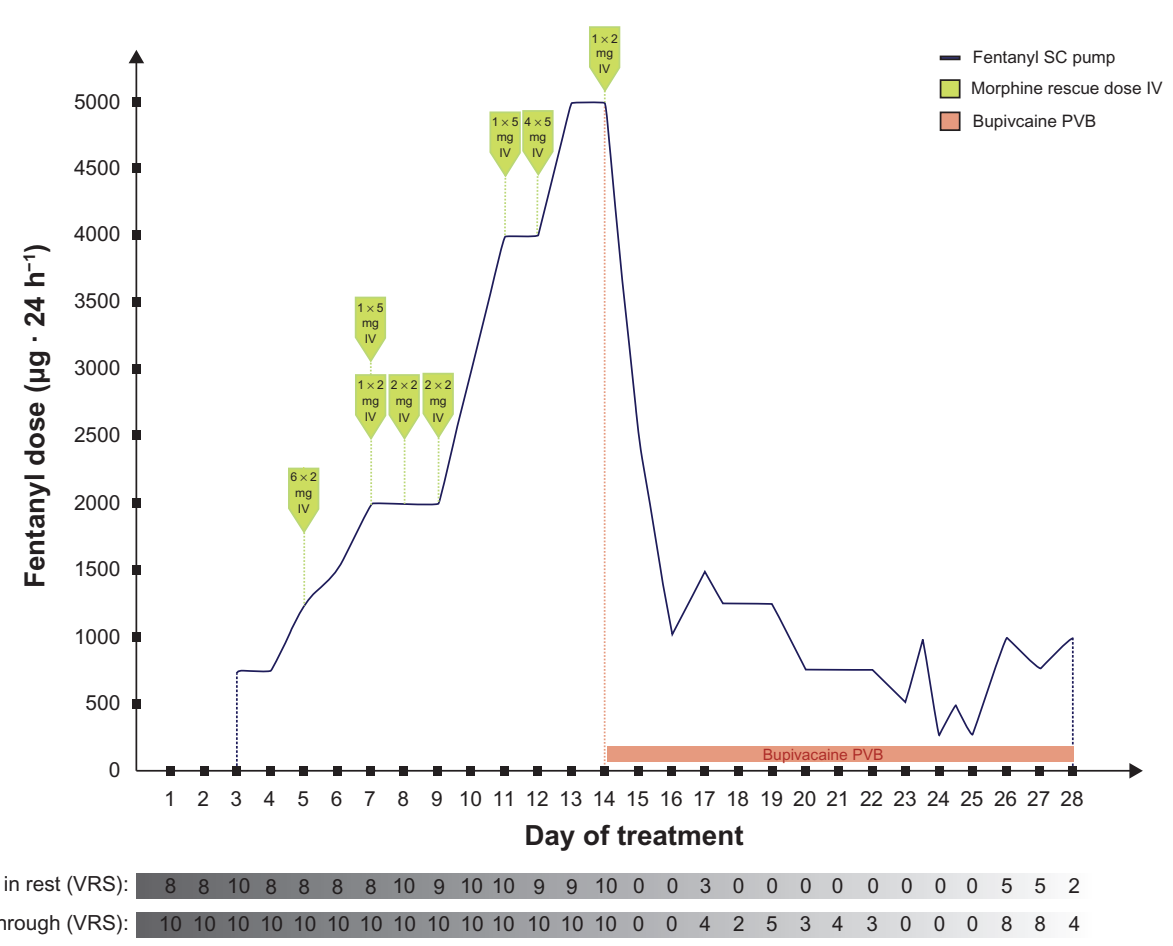

Figure I Drugs used for pain control during the stay in hospice in the patient presented.

Note: Blue line stands for fentanyl SC pump, morphine rescue doses are mapped out as green pointers, and bupivacaine paravertebral block is marked pink.

Abbreviations: IV, intravenous; PVB, paravertebral block; SC, subcutaneous; VRS, verbal rating scale; h, hours. 


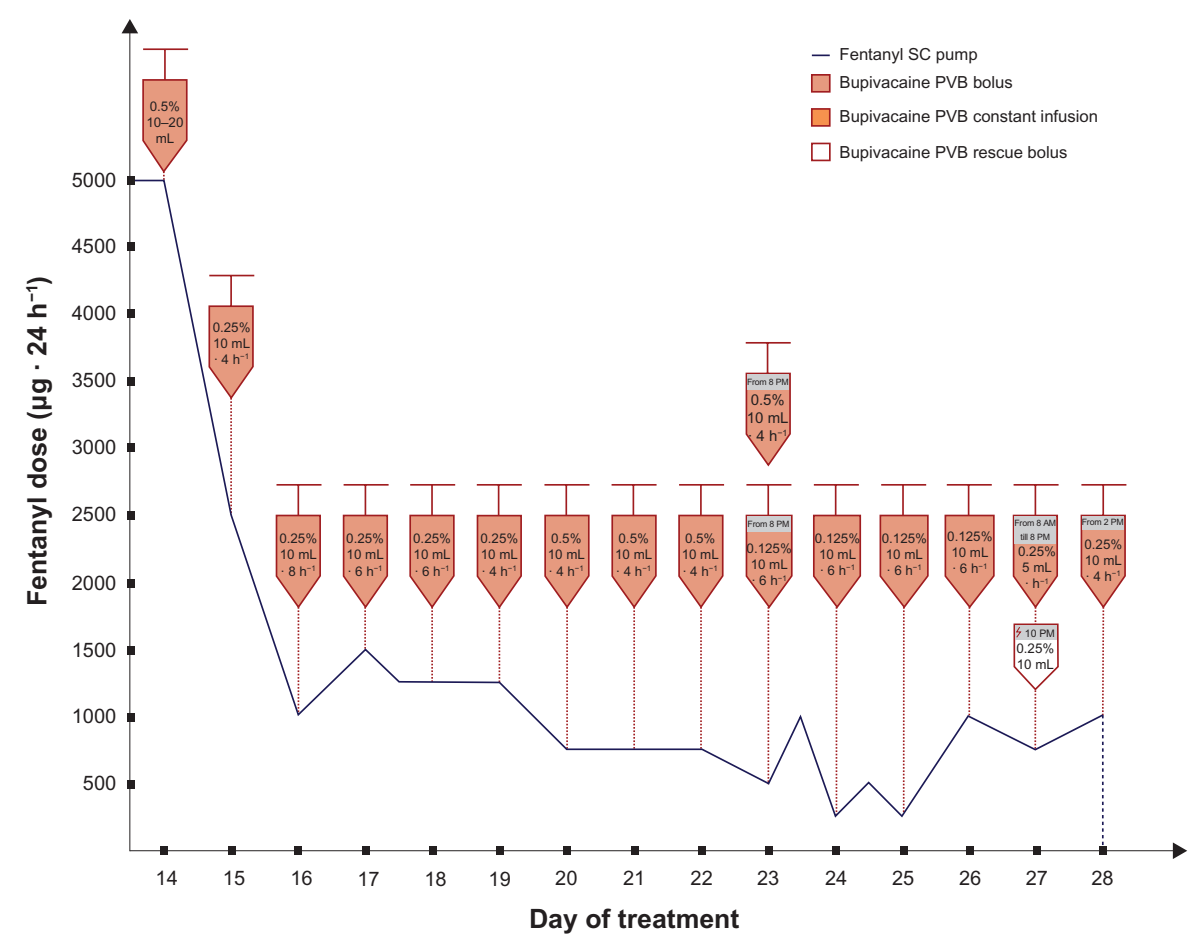

Figure 2 Doses of fentanyl in SC constant infusion and bupivacaine boluses administered paravertebrally to the patient.

Notes: Blue line stands for fentanyl SC pump, bupivacaine PVB boluses are marked pink, and bupivacaine PVB constant infusion is marked orange. Bupivacaine PVB rescue bolus is marked white with a purple frame.

Abbreviations: PVB, paravertebral block; SC, subcutaneous; h, hours.

$2.5-3.5 \mathrm{~cm}$ lateral to the midline at $\mathrm{L} 2$. Infiltration anesthesia with $1 \%-2 \%$ lignocaine solution was delivered to the place where the needle was subsequently inserted. Next, the Tuohy $18 \mathrm{G}$ needle (Smiths Group plc, London, UK) was inserted perpendicularly to the skin. Once the needle reached the transverse process it was slightly withdrawn and directed upwards. Having passed the transverse process, the needle was directed medially, with the needle bevel up (Figures 4 and 5). A catheter was inserted through the needle until resistance was felt. The needle was subsequently removed and the
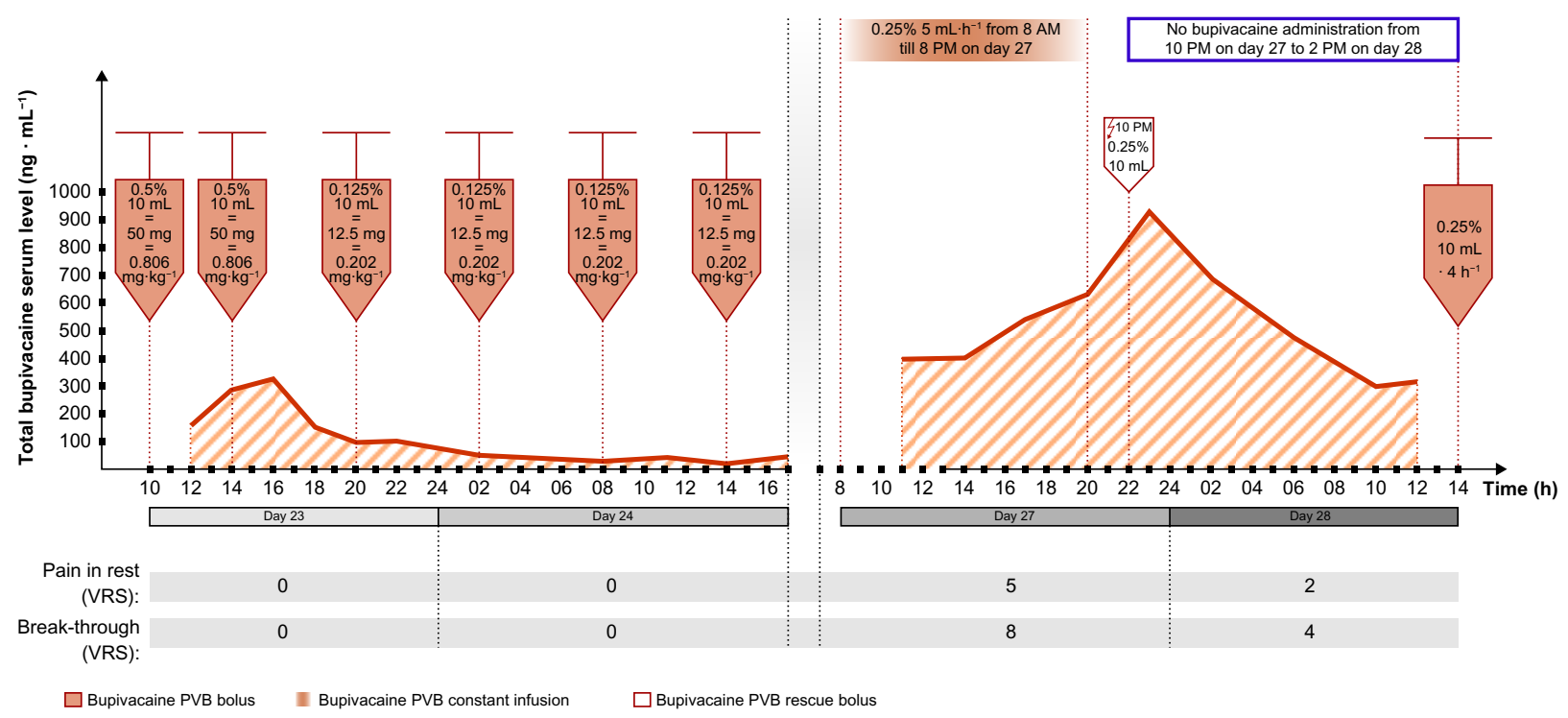

Figure 3 Total bupivacaine serum levels following its administration in boluses and in constant paravertebral infusion ( $\mathrm{ng} \cdot \mathrm{mL}^{-1}$ ) in the presented case.

Notes: Red line shows bupivacaine serum concentrations, bupivacaine PVB constant infusion is marked orange, and pink "syringes" stand for bupivacaine PVB boluses. Bupivacaine PVB rescue bolus is marked white with a purple frame.

Abbreviations: PVB, paravertebral block; VRS, verbal rating scale; $h$, hours. 


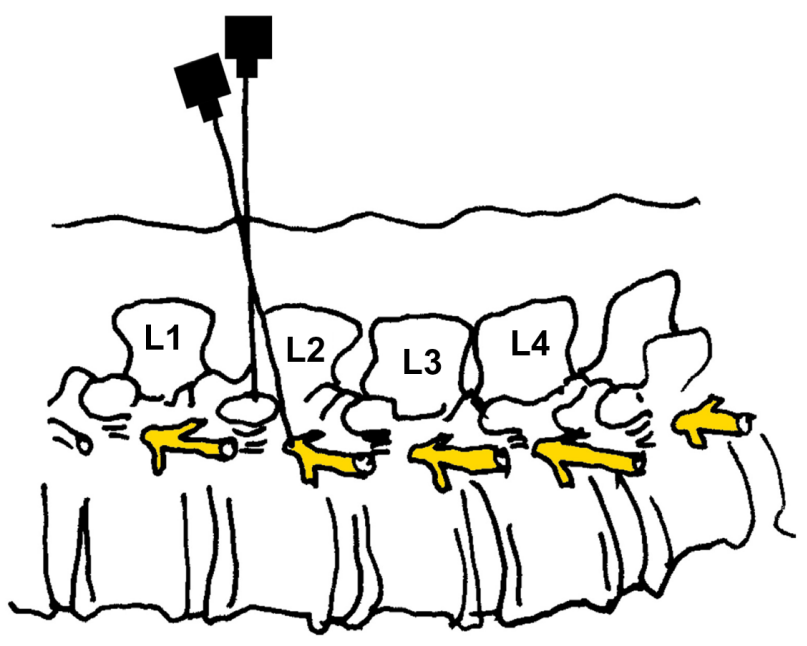

Figure 4 Paravertebral blockade (PVB): needle insertion, side view.

catheter was secured to the back (Figure 6). Next, repeated aspiration for blood and cerebrospinal fluid (CSF) was performed. The catheter was introduced at the L2 interspace. Following that, contrast media $\left(2 \mathrm{~mL}\right.$ of Ultravist ${ }^{\circledR} 370$ [lopromide]; Bayer AG, Leverkusen, Germany) were injected via the indwelling catheter. $\mathrm{L} 2$ lateroposterior radiograph was made to confirm the position of the catheter and evaluate the spread of the injected solution in the paravertebral space. Radiographs showed that the contrast solution was located in the posterolateral L2 space. (Figure 7). Then, $2 \mathrm{~mL}$ of $2 \%$

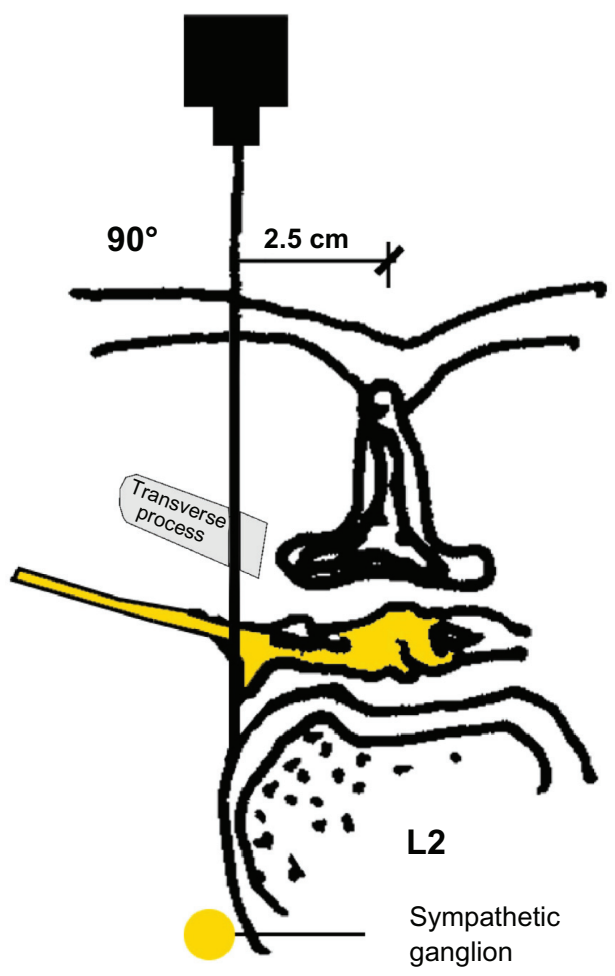

Figure 5 Paravertebral blockade (PVB): needle insertion, cross-section.

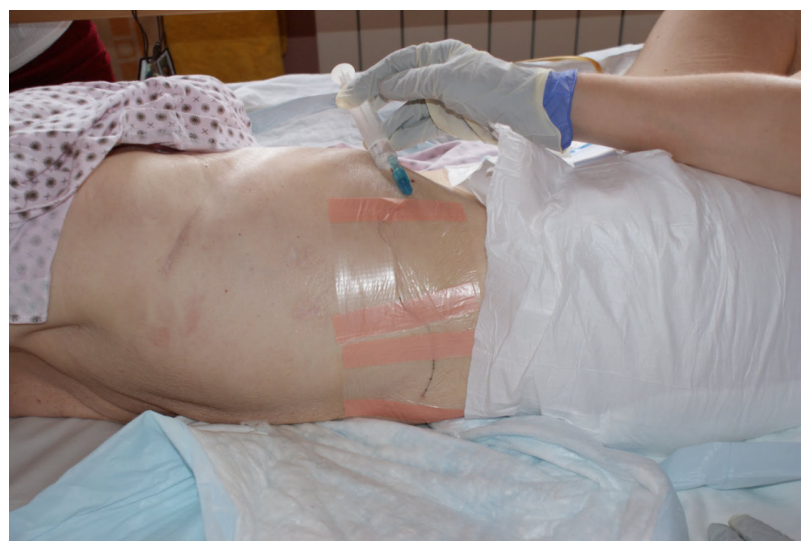

Figure 6 Paravertebral blockade (PVB): a catheter placement to the skin, the bolus of bupivacaine administration in the patient presented.

lidocaine was administered via the catheter, and no signs of lower extremity anesthesia (pain relief or decrease in muscle tone) were noticed during 5 minutes of observation. Next, $10 \mathrm{~mL} 0.5 \%$ bupivacaine hydrochloride (Bupivacaine; Claris Lifesciences Ltd, Ahmedabad, India) were administered paravertebrally via the same catheter. The patient reported effective, unilateral sensory analgesia: significant pain relief 20 minutes after the block placement. 20 minutes after the bupivacaine administration, the patient underwent analgesia level testing. A wet, cold gauze pad was moved from the chest to her lower extremity. When it reached the iliac crest, the patient stopped feeling cold on the blocked (right) side. The patient could move her right leg with no pain (active movements).

During the procedure, and after the first bupivacaine administration, no side effects and no complications were noticed. Blood pressure (BP) and heart rate (HR)

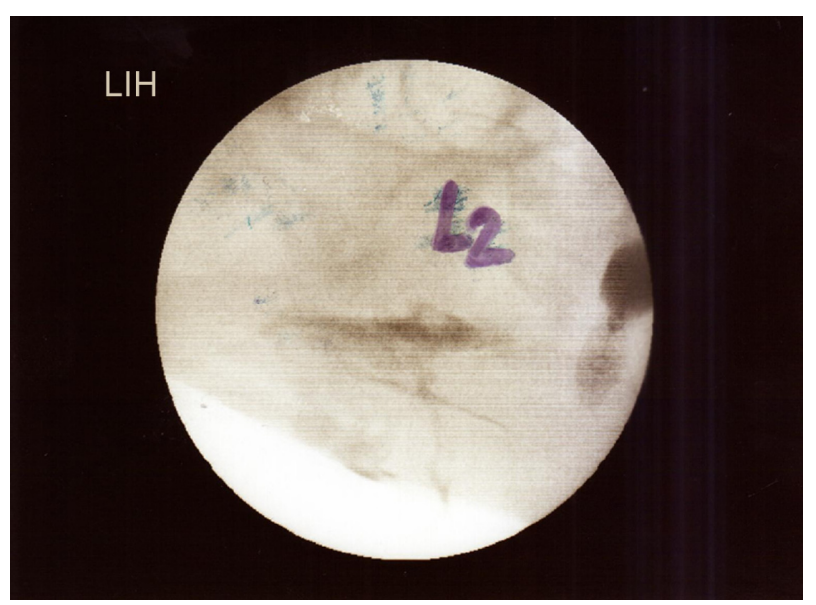

Figure 7 Radiograph in lateral projections obtained after the injection of contrast media to the paravertebral space in the presented patient $(2 \mathrm{~mL}$ of contrast solution is seen against the posterolateral aspect of the vertebral column). 
remained within the normal range. BP was measured only in lying position because of the patient's condition. Oxygen saturation before, during, soon after, and later after the PVB procedure ranged from $92 \%-96 \%$.

No signs of bupivacaine toxicity were demonstrated by the patient.

Electrocardiography (ECG) examination revealed no pathological changes caused by the bupivacaine administration.

\section{Bupivacaine serum level determination}

To determine serum bupivacaine levels, blood samples (2-3 mL per sample) from the central vein catheter (placed on day 14 , after the admission) were taken in 2-3 hourly intervals: just before and 2-3 hours after the bupivacaine paravertebral administration, on 3 separate days. Blood samples were centrifuged and serum was separated and stored at $-25^{\circ} \mathrm{C}$ before assay. Bupivacaine serum concentrations were determined using modified high-performance liquid chromatography-ultraviolet (HPLC-UV) method developed by Qin et al. ${ }^{35}$ The analysis was performed in HPLC system HP1 100 (Hewlett-Packard Company, Palo Alto, CA, USA) equipped with UV detector. $500 \mu \mathrm{L}$ of serum sample was spiked with papaverine (internal standard [IS]) solution with the final concentration of IS in matrix $200 \mathrm{ng} \cdot \mathrm{mL}^{-1}$. Samples were alkalized, using $100 \mu \mathrm{L}$ of $10 \%$ sodium carbonate aqueous solution, and extracted with $2 \mathrm{~mL}$ of $\mathrm{n}$-hexane. The organic layer was collected and evaporated to dryness at $40^{\circ} \mathrm{C}$ under a gentle stream of nitrogen. The residue was dissolved in 50 $\mu \mathrm{L}$ of methanol and $50 \mu \mathrm{L}$ of the mobile phase consisting of acetonitrile and $30 \mathrm{mM}$ potassium dihydrogen phosphate buffer (containing $0.16 \%$ triethylamine and adjusted to $\mathrm{pH}$ 4.9 with $1 \mathrm{M}$ orthophosphoric acid), mixed at the ratio of 25:75 (volume/volume). The analytes were resolved on RP18e column (LiChrospher, Merck Millipore, Billerica, MA, USA). The column temperature was fixed at $40^{\circ} \mathrm{C}$ and the mobile phase flow rate was $1 \mathrm{~mL} \cdot$ minutes $^{-1}$. The wavelength used for detection was $210 \mathrm{~nm}$ (0-9.5 minutes of the assay) for bupivacaine and $240 \mathrm{~nm}$ (9.5-13.5 minutes of the assay) for IS. Before the analysis of bupivacaine levels in the patient, the method was validated.

\section{Results}

Fentanyl SC constant infusion and MF rescue doses (2-5 mg IV) did not control the pain (according to VRS: pain of the right leg in rest equaled $7-10 / 10$ and the breakthrough pain was 10/10 during the first 13 days in hospice) (Figure 1). After the first dose of $10 \mathrm{~mL} 0.5 \%$ bupivacaine solution administration, the onset of analgesia was rapid (maximal analgesia after 20 minutes). Fifteen and 30 minutes after the paravertebral bupivacaine injection, the pain control was excellent (VRS for pain at rest and on leg movements equaled 0 ). The duration of analgesia was 11 hours. During the next 24 hours, bupivacaine doses $(10 \mathrm{~mL}$ of $0.5 \%$ solution) were given on demand (in 8-11 hour intervals). During the following days of hospitalization, the dose and/or intervals between the bupivacaine in boluses were titrated depending on satisfactory pain control $(10 \mathrm{~mL}$ of $0.125 \%-0.5 \%$ solution that equaled the doses $12.5-50 \mathrm{mg}$ ) (Figure 2). Fentanyl demand decreased substantially and no rescue MF dose was needed after bupivacaine boluses introduction, with satisfactory pain control (VRS: pain in rest equaled $0-3 / 10$, breakthrough pain equaled $0-5 / 10$ ), much less sedation, and decreased risk for other opioid side effects (Figure 1). This improved the patient's quality of life. Active leg movements in a supine position were possible. Next, constant infusion $(5 \mathrm{~mL}$ of $0.25 \%$ solution per hour $=12.5 \mathrm{mg} \cdot$ hours $^{-1}$ ) resulted in serum drug concentrations higher than caused by boluses, but the pain control was not satisfactory (Figure 3). Total dose of bupivacaine in PVB constant infusion was higher than the one given in boluses (median $150 \mathrm{mg} \cdot 12$ hours $^{-1}, 0.202 \mathrm{mg} \cdot \mathrm{kg}^{-1} \cdot$ hours $^{-1}$ and $68.75 \mathrm{mg} \cdot 12$ hours $^{-1}, 0.0924 \mathrm{mg} \cdot \mathrm{kg}^{-1} \cdot$ hours $^{-1}$, respectively). Bupivacaine serum concentrations, following its administrations in boluses, ranged from 22.9 to $573.5 \mathrm{ng} \cdot \mathrm{mL}^{-1}$ (median $99.7 \mathrm{ng} \cdot \mathrm{mL}^{-1}$ ). Constant paravertebral bupivacaine infusion caused serum drug levels to range from 396.9 to $634.1 \mathrm{ng} \cdot \mathrm{mL}^{-1}$ (median $477.5 \mathrm{ng} \cdot \mathrm{mL}^{-1}$ ). The bupivacaine bolus $(10 \mathrm{~mL}$ of $0.25 \%$ solution $=25 \mathrm{mg})$, given 2 hours after constant infusion withdrawal, caused a rise in its serum level up to $927.4 \mathrm{ng} \cdot \mathrm{mL}^{-1}$ (Figure 3). Bupivacaine's elimination constant was 0.262 hours $^{-1}$ and $t_{1 / 2}$ was 2.64 hours during treatment with the drug in boluses, while during constant bupivacaine infusion its elimination constant and $t_{1 / 2}$ were 0.089 hours $^{-1}$ and 7.8 hours, respectively.

\section{Discussion}

In the present case, PVB recommended for pain relief due to pathological fracture ${ }^{36}$ was simple to perform, had a low sideeffects profile, and led to fast, reliable pain relief, similarly to the observations of many other authors. ${ }^{1,12,21,26,37,38}$

The patient suffered from intractable pain despite constant SC fentanyl infusion (at different rates) and IV $\mathrm{MF}$ rescue doses. Because of inadequate pain control with morphine, its side effects, concomitant renal impairment, and SC fentanyl continuous infusion not being successful 
in giving the patient satisfactory pain relief, ED block was considered. PVB was finally introduced after taking into consideration the patient's INR value, concomitant heart insufficiency, and metastases to the bones and lungs followed by lobectomy. Systemic cancer disease is a dynamic process. In case of vertebral and epidural metastases (which may be undiagnosed in patients with prognoses $<3$ months in palliative care) PVB seems to be superior to ED or intrathecal drug application. The fact that PBV is followed by unilateral nerve blockade is an advantage. PVB seems to be less gravity-dependent than an ED or intrathecal block. The lower risk of paravertebral block's possible complications, mentioned above, compared with those of spinal or epidural anaesthesia was also taken into account. Introduction of paravertebral bupivacaine boluses resulted in excellent pain control, substantial decrease in fentanyl consumption, and MF rescue dose withdrawal. A similar effect was noticed by $\mathrm{Ng}$ et al: bupivacaine reduced MF consumption significantly in patients after hysterectomy. ${ }^{39}$ Better social interaction was undeniably beneficial. Postoperative acute and/or chronic pain is well ${ }^{40}$ or even better ${ }^{41}$ controlled by continuous bupivacaine infusion, than by boluses. Our observations do not confirm these remarks. LA should spread over a few dermatomes: L1-L4 in case of lower extremity analgesia (lumbar plexus). In the case described, boluses of $10 \mathrm{~mL}$ were more effective than continuous infusion, proving that the efficacy of peripheral nerve blockade in lumbar region depends not only on the dose of LA, but also on its volume. ${ }^{1}$ Systemic distribution of LAs may be life-threatening; their mode of administration and dosage should be optimized to provide reliable and safe treatment. PVB requires relatively high doses of LA. ${ }^{42}$ Systemic absorption of a LA is the fastest when injected intercostally, compared to other modes of regional administration. ${ }^{43,44}$ Predisposing factors for potential LAs' toxic effects are aging, small patient size, liver insufficiency, decreased hepatic blood flow (influenced by disease and/or drugs), hypovolemia, drug-drug interactions, renal impairment, hypoalbuminemia, hypocalcemia, ${ }^{45}$ extensive surgical disruption of tissue (pleurae) administered with LA, and inappropriate LA administration. ${ }^{46}$ Serious and fatal systemic toxicity of bupivacaine was described by Baaijens et $\mathrm{al}^{47}$ in cancer patients and Fagenholz et $\mathrm{al}^{46}$ after thoracotomy due to mycetoma. In cancer patients, numerous disturbances frequently coexist. Renal impairment was a contraindication to regular MF and caused slower drug elimination in the urine of the patient in the present case. Polytherapy could also influence drug elimination. According to Knudsen et al, bupivacaine IV infusion influences both systolic and diastolic left ventricular functions ${ }^{48}$ leading to decreased blood flow through the liver. In the case given, bupivacaine's systemic effect was avoided, but heart insufficiency and subsequent decreased hepatic blood flow could have affected specific drug clearance.

The patient had been also diagnosed with essential hypertension. BP and heart rate remained within recommended ranges during her stay in the hospice. The patient had hypoproteinemia, hypoalbuminemia, and hypocalcemia (Table 2); thus, after the decision of performing PVB with bupivacaine, which is eliminated unchanged in the urine in $\pm 1 \% 0^{43,49}$ and its hepatic extraction ratio value is $0.31^{50}$ (the lowest of the amide-type local anesthetics), the LA's dosage in the patient was still carefully chosen and reconsidered.

Bupivacaine protein binding is high $( \pm 95 \%)$. The drug is bound both to albumin (low affinity, high capacity site) and to $\alpha 1$-acid glycoprotein (high affinity, low capacity site). In cancer disease, concentrations of the latter are increased and may be responsible for the decrease in free drug fraction in contrast to the effect of hypoalbuminemia. ${ }^{51}$ In the patient presented, plasma $\alpha$-acid glycoprotein levels were increased and hypoalbuminemia was progressing during the course of hospitalization. Increased free drug fraction should have been eliminated faster renally, but was limited by reduced renal perfusion. The highest $\alpha 1$-acid glycoprotein concentration was detected during the constant bupivacaine infusion (Table 1). Bupivacaine's $t_{1 / 2}$ ( 7.8 hours), estimated from the drug serum levels on that day, reflects prolonged drug elimination when compared to the day of bolus administration $\left(t_{1 / 2}=2.64\right.$ hours $)$. Free bupivacaine concentrations were probably lower than could have been predicted from the dose administered and the actual albumin level: simultaneously elevated $\alpha 1$-acid glycoprotein bound a part of the free drug fraction. The elimination half-life of bupivacaine in boluses, close to population values, confirms the assumption. Elevated plasma $\alpha 1$-acid glycoprotein could have contributed to the protection against the systemic toxicity of bupivacaine and its slower hepatic clearance. ${ }^{52} \mathrm{~A}$ dynamic equilibrium between bound and unbound fractions of the drug is influenced by drug-drug interactions. Coadministered fentanyl could compete with bupivacaine for albumin and, to a lesser extent, for $\alpha 1$-acid glycoprotein binding. Fentanyl's protein binding $( \pm 85 \%)$ depends on albumin mainly in contrast to $\alpha 1$-acid glycoprotein (unusual among basic drugs).$^{53}$ While fentanyl's consumption decreased after bupivacaine introduction, a part of it was probably redistributed into the blood (three-compartment model of pharmacokinetics; following SC: $\mathrm{t}_{1 / 2}=7$ hours, approximately) from where it had been accumulated, ie, 
adipose tissue and muscle. Therefore, the importance of this pharmacokinetic interaction was decreased, but could have still played a role. Determination of unbound bupivacaine plasma levels would support these assumptions. Bupivacaine is metabolized by CYP3A4 isoenzyme of the P450 family. It was previously reported bupivacaine pharmacokinetics can be altered by coadministration of other CYP3A4 substrates. The coadministration of itraconazole, the CYP3A4 inhibitor, resulted in a bupivacaine-clearance decrease of $20 \%-25 \%{ }^{54}$ Therefore, it can be assumed that slower bupivacaine elimination, observed in the case after prolonged infusion, may be the consequence of the polytherapy applied. The patient received CYP3A4 substrates; fentanyl, midazolam, dexamethasone, and escitalopram. Bupivacaine serum concentrations after continuous infusion were much higher than after boluses and, due to limited enzyme capacity, the interactions might have become clinically relevant. ${ }^{54}$ LA absorption to the systemic circulation is dose- and route-dependent and determines the risk of their systemic toxicity. The correlation of serum bupivacaine levels and its anesthetic effect is poor (because of the variability of the drug distribution out of the administration site), but the drug's serum levels may indicate potential systemic toxic effects.

In patients with cancer disease, resistance to toxicity of bupivacaine was described. ${ }^{55}$

Bupivacaine toxic serum concentrations range from $2-4.5 \mathrm{mg} \cdot \mathrm{L}^{-1} .^{28,56}$ The threshold for early systemic toxicity is $2.5 \mathrm{mg} \cdot \mathrm{L}^{-1} \cdot{ }^{13}$ The threshold for CNS toxicity was observed at $2.25 \mathrm{mg} \cdot \mathrm{L}^{-1}$ in healthy volunteers, while it is slightly higher for cardiotoxicity ${ }^{57}$ and the drug serum concentrations below $3 \mathrm{mg} \cdot \mathrm{L}^{-1}$ are usually recommended. In the patient presented, total serum bupivacaine levels after the drug administration in boluses or in constant PVB infusion ranged from $0.023-0.927 \mathrm{mg} \cdot \mathrm{L}^{-1}$, remaining within safe concentration ranges. Fagenholz et $\mathrm{al}^{46}$ reported neurotoxicity in cachectic patients on antifungal therapy (voriconazole) after lobectomy. Because of extensive disruption of the pleurae and inappropriate bolus bupivacaine administration, the authors reported high serum drug concentration $\left(8 \mathrm{mg} \cdot \mathrm{L}^{-1}\right)$.

In the Cheung et al study, mean serum concentration of bupivacaine was $1.60 \mathrm{mg} \cdot \mathrm{L}^{-1}$ (and $>3 \mathrm{mg} \cdot \mathrm{L}^{-1}$ in three patients) at 30-48 hours following chronic extrapleural paravertebral drug infusion in children. The dosage was $1.25 \mathrm{mg} \cdot \mathrm{kg}^{-1}$ of $0.25 \%$ bupivacaine followed by $0.125 \%$ bupivacaine with adrenaline 1:400,000 at a rate of $0.2 \mathrm{~mL} \cdot \mathrm{kg}^{-1} \cdot$ hours ${ }^{-1}{ }^{22}$ Snowden et al found maximum levels of bupivacaine (plain and with epinephrine) ranging from $0.267-2.39 \mathrm{mg} \cdot \mathrm{mL}^{-1}$ (median $0.918 \mathrm{mg} \cdot \mathrm{mL}^{-1}$ ) and $0.479-1.08 \mathrm{mg} \cdot \mathrm{mL}^{-1}$ (median $\left.0.705 \mathrm{mg} \cdot \mathrm{mL}^{-1}\right)$, respectively, after its paravertebral bolus $\left(1 \mathrm{mg} \cdot \mathrm{kg}^{-1}\right)$ administration in adults. ${ }^{58}$ In the case presented, doses of bupivacaine in boluses ranged from $12.5-50 \mathrm{mg}$, resulting in its serum levels between 22.9 and $573.5 \mathrm{ng} \cdot \mathrm{mL}^{-1}$ (median $99.7 \mathrm{ng} \cdot \mathrm{mL}^{-1}$ ). Constant infusion of $12.5 \mathrm{mg} \cdot$ hour $^{-1}$ of the drug caused its serum concentrations to range from 396.9-634.1 $\mathrm{ng} \cdot \mathrm{mL}^{-1}$ (median $477.5 \mathrm{ng} \cdot \mathrm{mL}^{-1}$ ) with an increase up to $927.4 \mathrm{ng} \cdot \mathrm{mL}^{-1}$ entailed by extra dose, detected 5 hours after the bupivacaine withdrawal (Figure 3). Due to LA's systemic toxicity risk factors, bupivacaine's administration was transiently stopped before reintroducing drug administration in boluses. In most cases, systemic toxic effects from CNS appear prior to the cardiovascular ones. Coadministration of benzodiazepines, barbiturates or general anesthetics may mask CNS symptoms, and, in such a case, LA's cardiovascular toxicity may be observed first. The present patient received midazolam $\left(2.5-7.5 \mathrm{mg} \cdot 24\right.$ hours $\left.^{-1} \mathrm{SC}\right)$ or/and clonazepam $\left(0.5 \mathrm{mg} \cdot 24\right.$ hours $^{-1}$ po or SC). Estimation of bupivacaine serum levels was substantial to avoid its potential life-threatening systemic toxicity. In the present patient, calcium serum levels were lowered (Table 1). Hypocalcemia (ie, due to hypoalbuminemia, chronic renal failure, vitamin D deficiency, and $\mathrm{Mg}$ deficiency due to malabsorption; common in critically ill adults) predisposes to convulsions, hypotension, paresthesia, ECG changes, depression, confusion, and potentiates bupivacaine's neuro- and cardiotoxicity. ${ }^{45}$ Despite contributory factors for LA's systemic toxicity, the bupivacaine PVB in the present case was safe.

Lumbar PVB was confirmed to be a reliable, easy to perform method of analgesia in chronic, intractable pain of bone and neuropathic origin, even in a cancer patient with symptoms of opioid toxicity, contraindications to EDA, impaired renal function, hypoalbuminemia, and coadministered with the drugs affecting CYP3A4 isoenzyme (fentanyl, dexamethasone, midazolam, escitalopram). Total serum bupivacaine concentrations remained within safe ranges both during its administration in boluses and in constant paravertebral infusion. Unilateral PVB with bupivacaine in doses $<0.2625 \mathrm{mg} \cdot 24$ hours $^{-1}\left(<0.202 \mathrm{mg} \cdot \mathrm{kg}^{-1} \cdot\right.$ hours $\left.^{-1}\right)$ made active extremity movements possible in a supine position. However, in contrast to boluses, constant paravertebral infusion of bupivacaine was not fully effective in pain control, despite the use of higher doses and higher drug levels determined in serum. In the present patient, effectiveness of bupivacaine administered in boluses in the lumbar region was dose- and volume-dependent. In this case, the bupivacaine elimination half-life was prolonged during the constant paravertebral infusion as a consequence of bupivacaine's 
binding by acid $\alpha_{1}$-glycoprotein and more significant possible drug-drug interactions at higher drug concentrations.

It is important to note that PVB is considered as an equivalent for plexus or even ED block by some authors. Boezaart et al use the following terms interchangeably: lumbar paravertebral block; lumbar plexus block; and psoas compartment block. ${ }^{59}$ As they state, irrespective of their specific names, all paravertebral blocks share certain characteristics; they are performed just outside the dura matter that extends from the spinal cord dura and surrounds the roots of the nerves or plexuses. Therefore, the technique is, in principle, common for all the blocks, and the complications they may bring are also similar to the ones of an intrathecal or intramedullary injection. For this reason, all paravertebral blocks should be treated at the same level as the spinal epidural block. ${ }^{59}$

A lumbar plexus block may result from a psoas sheath block (drug administration in the space of the psoas sheath and into the muscle body of the psoas) or the psoas compartment block (drug administration behind the psoas sheath in the tissue plane between the psoas and quadratus, while the tissue plane mentioned is fornent and the lateral extension of the lumbar paravertebral space). A large volume of LA administered into the paravertebral space may be followed by the spread of the drug into the psoas compartment with resultant plexus block or, through intervertebral foramen, into epidural space. These consequences are volume- and pressure-dependent (LA injection under a higher pressure) ${ }^{60}$ In the case presented, the highest volume of bupivacaine administered paravertebrally equaled $10 \mathrm{~mL}$, and the potential spread of bupivacaine to the psoas space could contribute to the greater efficacy of boluses more than the highly dosed continuous bupivacaine infusion. The radiograph with contrast media in the paravertebral space does not confirm this assumption. In the case presented, proper catheter placing and spread of the injected solution in the paravertebral space was only confirmed via contrast media visualization in radio-examination (Figure 7) on the day of bupivacaine introduction.

\section{Acknowledgment}

Published with the written consent of the patient.

\section{Disclosure}

The authors report no conflicts of interest in this work.

\section{References}

1. Richardson J, Lonnqvist PA. Thoracic paravertebral block. Br J Anaesth. 1998;81:230-238.
2. Cheema SP, Ilsley D, Richardson J, Sabanathan S. A thermographic study of paravertebral analgesia. Anesthesia. 1995;50:118-121.

3. Tenicela R, Pollan SB. Paravertebral-peridural block technique: a unilateral thoracic block. Clin J Pain. 1990;6(3):227-234.

4. Moore DC. Anesthetic Techniques for Obstetrical Anesthesia and Analgesia. Springfield (IL): Charles C Thomas; 1964.

5. Eason MJ, Wyatt R. Paravertebral thoracic block-a reappraisal. Anesthesia. 1979;34:638-642.

6. Lonnqvist PA. Continous paravertebral block in children. Initial experience. Anesthesia. 1992;47(7):607-609.

7. Lonnqvist PA, Hesser U. Location of the paravertebral space in children and adolescents in relation to surface anatomy assessed by computer tomography. Pediatric Anesthesia. 1992;2:285-289.

8. Lonnqvist PA, Olsson GL. Paravertebral vs epidural block in children. Effects on postoperative morphine requirement after renal surgery. Acta Anaestesiol Scand. 1994;38:346-349.

9. Maguiar RV, Wheeler AS. Lumbar sympathetic block with bupivacaine: analgesia for labor. Anesth Analg. 1978;57:486-492.

10. Greengrass R, O’Brien F, Lyerly K, et al. Paravertebral block for breast cancer surgery. Can J Anaesth. 1996;43:858-861.

11. Jamieson BD, Mariano ER. Thoracic and lumbar paravertebral blocks for outpatient lithotripsy. J Clin Anesth. 2007;19:149-151.

12. Richardson J, Sabanathan S. Thoracic paravertebral analgesia. Acta Anaesthesiol Scand.1995;39:1005-1015.

13. Richardson J, Lonnqvist PA, Naja Z. Bilateral thoracic paravertebral block: potential and practice. Br J Anesth. 2011;106(2):164-171.

14. Richardson J, Sabanathan S, Mearns AJ, Shah RD, Goulden C. A prospective, randomised comparison of interpleural and paravertebral analgesia in thoracic surgery. Br J Anaesth. 1995;75:405-448.

15. Scarci M, Joshi A, Attia R. In patients undergoing thoracic surgery is paravertebral block as effective as epidural analgesia for pain management? Interact Cardiovasc Thorac Surg. 2010;10(1):92-96.

16. Williamson S, Kumar CM. Paravertebral block in head injured patient with chest trauma. Anesthesia. 1997;52:284-285.

17. Joshi GP, Bonnet F, Shah R, et al. A systematic review of randomised trials evaluating regional techniques for postthoracotomy analgesia. Anesth Analg. 2008;107(3):1026-1040.

18. Kairaluoma PM, Bachmann MS, Rosenberg PH, Pere PJ. Preincisional paravertebral block reduces the prevalence of chronic pain after breast burgery. Anesth Analg. 2006;103(3):703-708.

19. Kirvela O, Antila H. Thoracic paravertebral block in chronic postoperative pain. Reg Anesth. 1992;17:348-350.

20. Naccache N, Jabbour H, Nasser-Ayoub E, Abou Zeid H, Naja Z. Regional analgesia and breast cancer surgery. J Med Liban. 2009;57(2): 110-114.

21. Purcell-Jones G, Pither CE, Justins DM. Paravertebral somatic nerve block: a clinical, radiographic and computed tomographic study in chronic pain patients. Anesth Analg. 1989;68:32-39.

22. Cheung SL, Booker PD, Franks R, Pozi M. Serum concentrations of bupivacaine during prolong, continuous, paravertebral infusion in young infants. Br J Anaesth. 1997;79:9-13.

23. Antila $\mathrm{H}$, Kirveal O. Neurolytic paravertebral block in cancer pain. Acta Anaesthesiol Scand. 1998;42:581-585.

24. Bridenbaugh LD, Moore DC, Campbell DD. Management of upper abdominal cancer pain: treatment with celiac plexus block with alcohol. JAMA. 1964;190(10):877-880.

25. Lonnqvist PA, MacKenzie J, Soni AK, Conacher ID. Paravertebral blockade: failure rate and complications. Anesthesia. 1995;50:813-815.

26. Naja MZ, Lonnqvist PA. Somatic paraverebral nerve blockade: incidence of failed block and complications. Anesthesia. 2001;56:1184-1188.

27. Daly DJ, Myles PS. Update on the role of paravertebral blocks for thoracic surgery: are they worth it? Curr Opin Anaesthesiol. 2009;22(1): $38-43$.

28. Karmakar MK, Chui PT, Joynt GM, Ho AM. Thoracic paravertebral block for management of pain associated with multiple fractured ribs in patients with concomitant lumbar spinal trauma. Reg Anesth Pain Med. 2001;26:169-173. 
29. Davies RG, Myles PS, Graham JM. A comparison of the analgesic efficacy and side-effects of paravertebral vs epidural blockade for thoracotomy - a systematic review and meta-analysis of randomised trials. Br J Anaesth. 2007;99(5):768.

30. Richardson J, Sabanathan S, Jones J, Shah RD, Cheema S, Mearns AJ. A prospective, randomised comparison of preoperative and continuous balanced epidural or paravertebral bupivacaine on post thoracotomy pain, pulmonary function and stress responses. Br J Anaesth. 1999;83: 387-392.

31. Brown DL. Spinal, epidural and caudal anesthesia. In: Miller RD, editor. Anesthesia. 5th ed. New York: Churchill Livingstone Inc; 2000: 1491-1519.

32. Conacher ID, Kokri M. Postoperative paravertebral blocks for thoracic surgery: a radiological appraisal. Br J Anaesth. 1987;59:155-161.

33. Conacher ID. Resin injection of thoracic paravertebral spaces. $B r J$ Anaesth. 1988;61:657-661.

34. Saito T, Den S, Tanuma K, Tanuma Y, Carney E, Carlsson C. Anatomical bases for paravertebral anesthetic block: fluid communication between thoracic and lumbar paravertebral regions. Surg Radiol Anat. 1999;21;359-363.

35. Qin W, Jiao Z, Zhong M, et al. Simultaneous determination of procaine, lidocaine, ropivacaine, tetracaine and bupivacaine in human plasma by high performance liquid chromatography. J Chromatogr B Analyt Technol Biomed Life Sci. 2010;878:1185-1189.

36. Ripamonti CI, Santini D, Maranzano E, Berti M, Roila F; ESMO Guidelines Working Group. Management of cancer pain: ESMO Clinical Practice Guidelines. Ann Oncol. 2012;23 Suppl 7:139-154.

37. Cheema S, Richardson J, McGurgan P. Factors affecting the spread of bupivacaine in the adult paravertebral space. Anesthesia. 2003;58: 684-711.

38. Richardson J, Sabanathan S, Lonnqvist PA. Interpleural analgesia. Br J Anaesth. 1994;72:250.

39. Ng A, Swami A, Smith G, Davidson AC, Emembolu J. The analgesic effects of intraperitoneal and incisional bupivacaine with epinephrine after total abdominal hysterectomy. Anesth Analg. 2002;95(1): $158-162$.

40. Sabanathan S, Richardson J, Mearns AJ. Management of pain in thoracic surgery. Br J Hosp Med. 1993;50:114-120.

41. Catala E, Casas JI, Unzueta MC, Diaz X, Aliaga L, Villar Landeira JM. Continuous infusion is superior to bolus doses in thoracic paravertebral blocks after thoracotomies. J Cardiothorac Vasc Anesth. 1996;10(5): 586-588.

42. Kotze A, Scally A, Howell S. Efficacy and safety of different techniques of paravertebral block for analgesia after thoracotomy: asystematic review and metaregression. Br J Anaesth. 2009;103:626-636.

43. Rothstein P, Arthur GR, Feldman HS, Kopf GS, Covino BG. Bupivacaine for intercostal nerve blocks in children: blood concentrations and pharmacokinetics. Anesth Analg. 1986;65:625-632.
44. Willdeck-Lund G, Edstrom H. Etidocaine in intercostal nerve block for pain relief after thoracotomy; a comparison with bupivacaine. Acta Anaesthesiol Scand. 1975;60:33-38.

45. Aguilera IM, Vaughan RS. Calcium and the anaesthetist. Anesthesia. 2000;55:779-781.

46. Fagenholz PJ, Bowler GMR, Carnochan FM, Walker WS. Systemic local anaesthetic toxicity from continuous thoracic paravertebral block. Br J Anaesth. 2012;109(2):260-262.

47. Baaijens PF, Gielen MJ, Vree TB. Bupivacaine toxicity secondary to continuous cervical epidural infusion. Reg Anesth. 1995;20(2):163-168.

48. Knudsen K, Suurkula MB, Blomberg S. Central nervous and cardiovascular effects of iv infusions of ropivacaine, bupivacaine and placebo in volunteers. Br J Anaesth. 1997;78:507-514.

49. Baselt RC. Disposition of Toxic Drugs and Chemicals in Man. 7th ed. Foster City (CA): Biomedical Publications; 2004.

50. Tucker GT, Mather LE. Pharmacokinetics of local anaesthetics. $\mathrm{Br} J$ Anaesth. 1975;47:213-224.

51. Denson D, Coyle D, Thompson G, Myers J. Alpha1-acid glycoprotein and albumin in human serum bupivacaine binding. Clin Pharmacol Ther. 1984;35:409-415.

52. Meijer DK, Van der Sluijs P. The influence of binding to albumin and alpha 1-acid glycoprotein on the clearance of drugs by the liver. Pharm Weekbl Sci. 1987;9(2):65-74.

53. Wiesner G, Taeger K, Peter K. Serum protein binding of fentanyl. The effect of postoperative acute phase reaction with elevated alpha 1-acid glycoprotein and methodologic problems in determination by equilibrium dialysis. Anaesthesist. 1996;45(4):323-329.

54. Palkama VJ, Neuvonen PJ, Olkkola KT. Effect of itraconazole on the pharmacokinetics of bupivacaine enantiomers in healthy volunteers. Br J Anaesth. 1999;83(4):659-661.

55. Du Pen SL, Kharasch ED, Williams A, et al. Chronic epidural bupivacaine opioid infusion in intractable cancer pain. Pain. 1992;49: 293-300.

56. Berrisford RG, Sabanathan S, Mearns AJ, Clarke BJ, Hamdi A. Plasma concentrations of bupivacaine and its enantiomers during continuous extrapleural intercostal nerve block. Br JAnaesth. 1993;70: 201-204.

57. Bardsley H, Gristwood R, Baker H, Watson N, Nimmo W. A comparison of the cardiovascular effects of levobupivacaine and rac-bupivacaine following intravenous administration to healthy volunteers. $\mathrm{Br} J \mathrm{Clin}$ Pharmacol. 1998;46:245-429.

58. Snowden CP, Bower S, Conacher I. Plasma bupivacaine levels in paravertebral blockade in adults. Anesthesia. 1994;49:546.

59. Boezaart AP, Lucas SD, Elliot CE. Paravertebral block: cervical, thoracic, lumbar and sacral. Curr Opin Anaesthesiol. 2009;22(5):637-643.

60. Gadsden JC, Lindenmuth DM, Hadzic A, Xu D, Somasundarum L, Flisinski KA. Lumbar plexus block using high-pressure injection leads to contralateral and epidural spread. Anesthesiology. 2008;109:683-688.
OncoTargets and Therapy

\section{Publish your work in this journal}

OncoTargets and Therapy is an international, peer-reviewed, open access journal focusing on the pathological basis of all cancers, potential targets for therapy and treatment protocols employed to improve the management of cancer patients. The journal also focuses on the impact of management programs and new therapeutic agents and protocols on
Dovepress

patient perspectives such as quality of life, adherence and satisfaction. The manuscript management system is completely online and includes a very quick and fair peer-review system, which is all easy to use. Visit http://www.dovepress.com/testimonials.php to read real quotes from published authors. 